\title{
Extremal selections of multifunctions generating a continuous flow
}

\author{
by Alberto Bressan and Graziano Crasta (Trieste)
}

Abstract. Let $F:[0, T] \times \mathbb{R}^{n} \rightarrow 2^{\mathbb{R}^{n}}$ be a continuous multifunction with compact, not necessarily convex values. In this paper, we prove that, if $F$ satisfies the following Lipschitz Selection Property:

(LSP) For every $t, x$, every $y \in \overline{\mathrm{Co}} F(t, x)$ and $\varepsilon>0$, there exists a Lipschitz selection $\phi$ of $\overline{\mathrm{co}} F$, defined on a neighborhood of $(t, x)$, with $|\phi(t, x)-y|<\varepsilon$,

then there exists a measurable selection $f$ of $\operatorname{ext} F$ such that, for every $x_{0}$, the Cauchy problem

$$
\dot{x}(t)=f(t, x(t)), \quad x(0)=x_{0},
$$

has a unique Carathéodory solution, depending continuously on $x_{0}$.

We remark that every Lipschitz multifunction with compact values satisfies (LSP). Another interesting class for which (LSP) holds consists of those continuous multifunctions $F$ whose values are compact and have convex closure with nonempty interior.

1. Introduction. Let $F:[0, T] \times \mathbb{R}^{n} \rightarrow 2^{\mathbb{R}^{n}}$ be a continuous multifunction with compact, not necessarily convex values. If $F$ is Lipschitz continuous, it was shown in [5] that there exists a measurable selection $f$ of $F$ such that, for every $x_{0}$, the Cauchy problem

$$
\dot{x}(t)=f(t, x(t)), \quad x(0)=x_{0},
$$

has a unique Carathéodory solution, depending continuously on $x_{0}$.

In this paper, we prove that the above selection $f$ can be chosen so that $f(t, x) \in \operatorname{ext} F(t, x)$ for all $t, x$. More generally, the result remains valid if $F$ satisfies the following Lipschitz Selection Property:

(LSP) For every $t, x$, every $y \in \overline{\mathrm{co}} F(t, x)$ and $\varepsilon>0$, there exists a Lipschitz selection $\phi$ of $\overline{\mathrm{CO}} F$, defined on a neighborhood of $(t, x)$, with $|\phi(t, x)-y|<\varepsilon$.

1991 Mathematics Subject Classification: Primary 34A60.

Key words and phrases: differential inclusion, extremal selection. 
We remark that, by $[10,12]$, every Lipschitz multifunction with compact values satisfies (LSP). Another interesting class for which (LSP) holds consists of those continuous multifunctions $F$ whose values are compact and have convex closure with nonempty interior. Indeed, for any given $t, x, y, \varepsilon$, choosing $y^{\prime} \in \operatorname{int} \overline{\operatorname{co}} F(t, x)$ with $\left|y^{\prime}-y\right|<\varepsilon$, the constant function $\phi \equiv y^{\prime}$ is a local selection from $\overline{\mathrm{c}} F$ satisfying the requirements.

In the following, $\Omega \subseteq \mathbb{R}^{n}$ is an open set, $\bar{B}(0, M)$ is the closed ball centered at the origin with radius $M, \bar{B}(D, M T)$ is the closed neighborhood of radius $M T$ around the set $D$, while $\mathcal{A C}$ is the Sobolev space of all absolutely continuous functions $u:[0, T] \rightarrow \mathbb{R}^{n}$, with norm $\|u\|_{\mathcal{A C}}=$ $\int_{0}^{T}(|u(t)|+|\dot{u}(t)|) d t$.

THEOREM 1. Let $F:[0, T] \times \Omega \rightarrow 2^{\mathbb{R}^{n}}$ be a bounded continuous multifunction with compact values, satisfying (LSP). Assume that $F(t, x) \subseteq \bar{B}(0, M)$ for all $t, x$ and let $D$ be a compact set such that $\bar{B}(D, M T) \subset \Omega$. Then there exists a measurable function $f$ with

$$
f(t, x) \in \operatorname{ext} F(t, x) \quad \forall t, x,
$$

such that, for every $\left(t_{0}, x_{0}\right) \in[0, T] \times D$, the Cauchy problem

$$
\dot{x}(t)=f(t, x(t)), \quad x\left(t_{0}\right)=x_{0}
$$

has a unique Carathéodory solution $x(\cdot)=x\left(\cdot, t_{0}, x_{0}\right)$ on $[0, T]$, depending continuously on $t_{0}, x_{0}$ in the norm of $\mathcal{A C}$.

Moreover, if $\varepsilon_{0}>0$ and a Lipschitz continuous selection $f_{0}$ of $\overline{\mathrm{co}} F$ are given, then one can construct $f$ with the following additional property: Denoting by $y\left(\cdot, t_{0}, x_{0}\right)$ the unique solution of

$$
\dot{y}(t)=f_{0}(t, y(t)), \quad y\left(t_{0}\right)=x_{0},
$$

for every $\left(t_{0}, x_{0}\right) \in[0, T] \times D$ one has

$$
\left|y\left(t, t_{0}, x_{0}\right)-x\left(t, t_{0}, x_{0}\right)\right| \leq \varepsilon_{0} \quad \forall t \in[0, T] .
$$

The proof of the above theorem, given in Section 3, starts with the construction of a sequence $f_{n}$ of directionally continuous selections from $\overline{\mathrm{cO}} F$ which are piecewise Lipschitz continuous in the $(t, x)$-space. For every $u$ : $[0, T] \rightarrow \mathbb{R}^{n}$ in a class of Lipschitz continuous functions, we then show that the composed maps $t \rightarrow f_{n}(t, u(t))$ form a Cauchy sequence in $\mathcal{L}^{1}\left([0, T] ; \mathbb{R}^{n}\right)$ converging pointwise almost everywhere to a map of the form $f(\cdot, u(\cdot))$, taking values within the extreme points of $F$. This convergence is obtained through an argument which is considerably different from previous works. Indeed, it relies on a careful use of the likelihood functional introduced in [4], interpreted here as a measure of "oscillatory nonconvergence" of a set of derivatives. 
Among various corollaries, Theorem 1 yields an extension, valid for the wider class of multifunctions with the property (LSP), of the following results, proved in [7], [5] and [8], respectively.

(i) Existence of selections from the solution set of a differential inclusion, depending continuously on the initial data.

(ii) Existence of selections from a multifunction, which generate a continuous flow.

(iii) Contractibility of the solution sets of $\dot{x} \in F(t, x)$ and $\dot{x} \in \operatorname{ext} F(t, x)$.

These consequences, together with an application to bang-bang feedback controls, are described in Section 4. Topological properties of the set of solutions of nonconvex differential inclusions have been studied in $[3,6]$ with the technique of directionally continuous selections and in $[8,9,13]$ using the method of Baire category.

2. Preliminaries. As customary, $\bar{A}$ and $\overline{\mathrm{co}} A$ denote here the closure and the closed convex hull of $A$ respectively, while $A \backslash B$ indicates a settheoretic difference. The Lebesgue measure of a set $J \subset \mathbb{R}$ is $m(J)$. The characteristic function of a set $A$ is written as $\chi_{A}$.

In the following, $\mathcal{K}_{n}$ denotes the family of all nonempty compact convex subsets of $\mathbb{R}^{n}$, endowed with Hausdorff metric. A key technical tool used in our proofs will be the function $h: \mathbb{R}^{n} \times \mathcal{K}_{n} \rightarrow \mathbb{R} \cup\{-\infty\}$ defined by

$$
\begin{aligned}
& h(y, K) \\
& \quad \doteq \sup \left\{\left(\int_{0}^{1}|w(\xi)-y|^{2} d \xi\right)^{1 / 2} ; w:[0,1] \rightarrow K, \int_{0}^{1} w(\xi) d \xi=y\right\}
\end{aligned}
$$

with the understanding that $h(y, K)=-\infty$ if $y \notin K$. Observe that $h^{2}(y, K)$ can be interpreted as the maximum variance among all random variables supported inside $K$ whose mean value is $y$. The following results were proved in $[4]$ :

LEMMA 1. The map $(y, K) \mapsto h(y, K)$ is upper semicontinuous in both variables; for each fixed $K \in \mathcal{K}_{n}$ the function $y \mapsto h(y, K)$ is strictly concave down on $K$. Moreover, one has

$$
\begin{gathered}
h(y, K)=0 \quad \text { if and only if } y \in \operatorname{ext} K, \\
h^{2}(y, K) \leq r^{2}(K)-|y-c(K)|^{2},
\end{gathered}
$$

where $c(K)$ and $r(K)$ denote the Chebyshev center and the Chebyshev radius of $K$, respectively.

Remark 1. By the above lemma, the function $h$ has all the qualitative properties of the Choquet function $d_{F}$ considered, for example, in $[9$, 
Proposition 2.6]. It could thus be used within any argument based on Baire category. Moreover, the likelihood functional

$$
L(u) \doteq\left(\int_{0}^{T} h^{2}(\dot{u}(t), F(t, u(t))) d t\right)^{1 / 2}
$$

provides an upper bound to the distance $\|\dot{v}-\dot{u}\|_{\mathcal{L}^{2}}$ between derivatives, for solutions of $\dot{v} \in F(t, v)$ which remain close to $u$ uniformly on $[0, T]$. This additional quantitative property of the function $h$ will be a crucial ingredient in our proof.

For the basic theory of multifunctions and differential inclusions we refer to [1]. As in [2], given a map $g:[0, T] \times \Omega \rightarrow \mathbb{R}^{n}$, we say that $g$ is directionally continuous along the directions of the cone $\Gamma^{N}=\{(s, y) ;|y| \leq N s\}$ if

$$
g(t, x)=\lim _{k \rightarrow \infty} g\left(t_{k}, x_{k}\right)
$$

for every $(t, x)$ and every sequence $\left(t_{k}, x_{k}\right)$ in the domain of $g$ such that $t_{k} \rightarrow t$ and $\left|x_{k}-x\right| \leq N\left(t_{k}-t\right)$ for every $k$. Equivalently, $g$ is $\Gamma^{N}$-continuous iff it is continuous w.r.t. the topology generated by the family of all half-open cones of the form

$$
\{(s, y) ; \widehat{t} \leq s<\widehat{t}+\varepsilon,|y-\widehat{x}| \leq N(s-t)\}
$$

with $(\widehat{t}, \widehat{x}) \in \mathbb{R} \times \mathbb{R}^{n}, \varepsilon>0$. A set of the form (2.4) will be called an $N$-cone.

Under the assumptions on $\Omega, D$ made in Theorem 1, consider the set of Lipschitzean functions

$$
Y \doteq\{u:[0, T] \rightarrow \bar{B}(D, M T) ;|u(t)-u(s)| \leq M|t-s| \forall t, s\} .
$$

The Picard operator of a map $g:[0, T] \times \Omega \rightarrow \mathbb{R}^{n}$ is defined as

$$
\mathcal{P}^{g}(u)(t) \doteq \int_{0}^{t} g(s, u(s)) d s, \quad u \in Y .
$$

The distance between two Picard operators will be measured by

$$
\begin{aligned}
& \left\|\mathcal{P}^{f}-\mathcal{P}^{g}\right\| \\
& \quad=\sup \left\{\left|\int_{0}^{t}[f(s, u(s))-g(s, u(s))] d s\right| ; t \in[0, T], u \in Y\right\} .
\end{aligned}
$$

The next lemma will be useful in order to prove the uniqueness of solutions of the Cauchy problems (1.2).

LEMMA 2. Let $f$ be a measurable map from $[0, T] \times \Omega$ into $\bar{B}(0, M)$, with $\mathcal{P}^{f}$ continuous on $Y$. Let $D$ be compact, with $\bar{B}(D, M T) \subset \Omega$, and assume that the Cauchy problem

$$
\dot{x}(t)=f(t, x(t)), \quad x\left(t_{0}\right)=x_{0}, \quad t \in[0, T],
$$

has a unique solution, for each $\left(t_{0}, x_{0}\right) \in[0, T] \times D$. 
Then, for every $\varepsilon>0$, there exists $\delta>0$ with the following property. If $g:[0, T] \times \Omega \rightarrow \bar{B}(0, M)$ satisfies $\left\|\mathcal{P}^{g}-\mathcal{P}^{f}\right\| \leq \delta$, then for every $\left(t_{0}, x_{0}\right) \in$ $[0, T] \times D$, any solution of the Cauchy problem

$$
\dot{y}(t)=g(t, y(t)), \quad y\left(t_{0}\right)=x_{0}, \quad t \in[0, T],
$$

has distance $<\varepsilon$ from the corresponding solution of (2.6). In particular, the solution set of $(2.7)$ has diameter $\leq 2 \varepsilon$ in $\mathcal{C}^{0}\left([0, T] ; \mathbb{R}^{n}\right)$.

Proof. If the conclusion fails, then there exist sequences of times $t_{\nu}, t_{\nu}^{\prime}$, maps $g_{\nu}$ with $\left\|\mathcal{P}^{g_{\nu}}-\mathcal{P}^{f}\right\| \rightarrow 0$, and couples of solutions $x_{\nu}, y_{\nu}:[0, T] \rightarrow$ $\bar{B}(D, M T)$ of

$$
\dot{x}_{\nu}(t)=f\left(t, x_{\nu}(t)\right), \quad \dot{y}_{\nu}(t)=g_{\nu}\left(t, y_{\nu}(t)\right), \quad t \in[0, T],
$$

$$
x_{\nu}\left(t_{\nu}\right)=y_{\nu}\left(t_{\nu}\right) \in D, \quad\left|x_{\nu}\left(t_{\nu}^{\prime}\right)-y_{\nu}\left(t_{\nu}^{\prime}\right)\right| \geq \varepsilon \quad \forall \nu .
$$

By taking subsequences, we can assume that $t_{\nu} \rightarrow t_{0}, t_{\nu}^{\prime} \rightarrow \tau, x_{\nu}\left(t_{0}\right) \rightarrow x_{0}$, while $x_{\nu} \rightarrow x$ and $y_{\nu} \rightarrow y$ uniformly on $[0, T]$. From (2.8) it follows that

$$
\begin{gathered}
\left|y(t)-x_{0}-\int_{t_{0}}^{t} f(s, y(s)) d s\right| \leq\left|y(t)-y_{\nu}(t)\right|+\left|x_{0}-y_{\nu}\left(t_{0}\right)\right| \\
+\left|\int_{t_{0}}^{t}\left[f(s, y(s))-f\left(s, y_{\nu}(s)\right)\right] d s\right|+\left|\int_{t_{0}}^{t}\left[f\left(s, y_{\nu}(s)\right)-g_{\nu}\left(s, y_{\nu}(s)\right)\right] d s\right| .
\end{gathered}
$$

As $\nu \rightarrow \infty$, the right hand side of (2.10) tends to zero, showing that $y(\cdot)$ is a solution of (2.6). By the continuity of $\mathcal{P}^{f}, x(\cdot)$ is also a solution of $(2.6)$, distinct from $y(\cdot)$ because

$$
|x(\tau)-y(\tau)|=\lim _{\nu \rightarrow \infty}\left|x_{\nu}(\tau)-y_{\nu}(\tau)\right|=\lim _{\nu \rightarrow \infty}\left|x_{\nu}\left(t_{\nu}^{\prime}\right)-y_{\nu}\left(t_{\nu}^{\prime}\right)\right| \geq \varepsilon .
$$

This contradicts the uniqueness assumption, proving the lemma.

3. Proof of the main theorem. Observing that ext $F(t, x)=$ ext $\overline{c o} F(t, x)$ for every compact set $F(t, x)$, it is clearly not restrictive to prove Theorem 1 under the additional assumption that all values of $F$ are convex. Moreover, the bounds on $F$ and $D$ imply that no solution of the Cauchy problem

$$
\dot{x}(t) \in F(t, x(t)), \quad x\left(t_{0}\right)=x_{0}, \quad t \in[0, T],
$$

with $x_{0} \in D$, can escape from the set $\bar{B}(D, M T)$. Therefore, it suffices to construct the selection $f$ on the compact set $\Omega^{\dagger} \doteq[0, T] \times \bar{B}(D, M T)$. Finally, since every convex-valued multifunction satisfying (LSP) admits a globally defined Lipschitz selection, it suffices to prove the second part of the theorem, with $f_{0}$ and $\varepsilon_{0}>0$ assigned. 
We shall define a sequence of directionally continuous selections of $F$, converging a.e. to a selection from ext $F$. The basic step of our constructive procedure will be provided by the next lemma.

Lemma 3. Fix any $\varepsilon>0$. Let $S$ be a compact subset of $[0, T] \times \Omega$ and let $\phi: S \rightarrow \mathbb{R}^{n}$ be a continuous selection of $F$ such that

$$
h(\phi(t, x), F(t, x))<\eta \quad \forall(t, x) \in S,
$$

with $h$ as in (2.1). Then there exists a piecewise Lipschitz selection $g: S \rightarrow$ $\mathbb{R}^{n}$ of $F$ with the following properties:

(i) There exists a finite covering $\left\{\Gamma_{i}\right\}_{i=1, \ldots, \nu}$, consisting of $\Gamma^{M+1}$-cones, such that, if we define the pairwise disjoint sets $\Delta^{i} \doteq \Gamma_{i} \backslash \bigcup_{l<i} \Gamma_{l}$, then on each $\Delta^{i}$ the following holds:

(a) There exist Lipschitzean selections $\psi_{j}^{i}: \overline{\Delta^{i}} \rightarrow \mathbb{R}^{n}, j=0, \ldots, n$, such that

$$
\left.g\right|_{\Delta^{i}}=\sum_{j=0}^{n} \psi_{j}^{i} \chi_{A_{j}^{i}},
$$

where each $A_{j}^{i}$ is a finite union of strips of the form $\left(\left[t^{\prime}, t^{\prime \prime}\right) \times \mathbb{R}^{n}\right)$ $\cap \Delta^{i}$.

(b) For every $j=0, \ldots, n$ there exists an affine $\operatorname{map} \varphi_{j}^{i}(\cdot)=\left\langle a_{j}^{i}, \cdot\right\rangle+b_{j}^{i}$ such that

$$
\varphi_{j}^{i}\left(\psi_{j}^{i}(t, x)\right) \leq \varepsilon, \varphi_{j}^{i}(z) \geq h(z, F(t, x)), \quad \forall(t, x) \in \overline{\Delta^{i}}, z \in F(t, x) .
$$

(ii) For every $u \in Y$ and every interval $\left[\tau, \tau^{\prime}\right]$ such that $(s, u(s)) \in S$ for $\tau \leq s<\tau^{\prime}$, the following estimates hold:

$$
\begin{gathered}
\left|\int_{\tau}^{\tau^{\prime}}[\phi(s, u(s))-g(s, u(s))] d s\right| \leq \varepsilon, \\
\int_{\tau}^{\tau^{\prime}}|\phi(s, u(s))-g(s, u(s))| d s \leq \varepsilon+\eta\left(\tau^{\prime}-\tau\right) .
\end{gathered}
$$

R e mark 2. Thinking of $h(y, K)$ as a measure for the distance of $y$ from the extreme points of $K$, the above lemma can be interpreted as follows. Given any selection $\phi$ of $F$, one can find a $\Gamma^{M+1}$-continuous selection $g$ whose values lie close to the extreme points of $F$ and whose Picard operator $\mathcal{P}^{g}$, by (3.4), is close to $\mathcal{P}^{\phi}$. Moreover, if the values of $\phi$ are near the extreme points of $F$, i.e. if $\eta$ in (3.1) is small, then $g$ can be chosen close to $\phi$. The estimate (3.5) will be a direct consequence of the definition (2.1) of $h$ and of Hölder's inequality. 
Re mark 3. Since $h$ is only upper semicontinuous, the two assumptions $y_{\nu} \rightarrow y$ and $h\left(y_{\nu}, K\right) \rightarrow 0$ do not necessarily imply $h(y, K)=0$. As a consequence, the a.e. limit of a convergent sequence of approximately extremal selections $f_{\nu}$ of $F$ need not take values inside ext $F$. To overcome this difficulty, the estimates in (3.3) provide upper bounds for $h$ in terms of the affine maps $\varphi_{j}^{i}$. Since each $\varphi_{j}^{i}$ is continuous, limits of the form $\varphi_{j}^{i}\left(y_{\nu}\right) \rightarrow \varphi_{j}^{i}(y)$ will be straightforward.

Proof of Lemma 3 . For every $(t, x) \in S$ there exist values $y_{j}(t, x)$ $\in F(t, x)$ and coefficients $\theta_{j}(t, x) \geq 0$ with

$$
\begin{gathered}
\phi(t, x)=\sum_{j=0}^{n} \theta_{j}(t, x) y_{j}(t, x), \quad \sum_{j=0}^{n} \theta_{j}(t, x)=1, \\
h\left(y_{j}(t, x), F(t, x)\right)<\varepsilon / 2 .
\end{gathered}
$$

By the concavity and the upper semicontinuity of $h$, for every $j=0, \ldots, n$ there exists an affine function $\varphi_{j}^{(t, x)}(\cdot)=\left\langle a_{j}^{(t, x)}, \cdot\right\rangle+b_{j}^{(t, x)}$ such that

$$
\begin{gathered}
\varphi_{j}^{(t, x)}\left(y_{j}(t, x)\right)<h\left(y_{j}(t, x), F(t, x)\right)+\varepsilon / 2<\varepsilon, \\
\varphi_{j}^{(t, x)}(z)>h(z, F(t, x)) \quad \forall z \in F(t, x) .
\end{gathered}
$$

By (LSP) and the continuity of each $\varphi_{j}^{(t, x)}$, there exists a neighborhood $\mathcal{U}$ of $(t, x)$ together with Lipschitzean selections $\psi_{j}^{(t, x)}: \mathcal{U} \rightarrow \mathbb{R}^{n}$ such that, for every $j$ and every $(s, y) \in \mathcal{U}$,

$$
\begin{aligned}
\left|\psi_{j}^{(t, x)}(s, y)-y_{j}(t, x)\right| & <\frac{\varepsilon}{4 T}, \\
\varphi_{j}^{(t, x)}\left(\psi_{j}^{(t, x)}(s, y)\right) & <\varepsilon .
\end{aligned}
$$

Using again the upper semicontinuity of $h$, we can find a neighborhood $\mathcal{U}^{\prime}$ of $(t, x)$ such that

$$
\varphi_{j}^{(t, x)}(z) \geq h(z, F(s, y)) \quad \forall z \in F(s, y),(s, y) \in \mathcal{U}^{\prime}, j=0, \ldots, n .
$$

Choose a neighborhood $\Gamma_{t, x}$ of $(t, x)$, contained in $\mathcal{U} \cap \mathcal{U}^{\prime}$, such that, for every point $(s, y)$ in the closure $\bar{\Gamma}_{t, x}$, one has

$$
|\phi(s, y)-\phi(t, x)|<\frac{\varepsilon}{4 T} .
$$

It is not restrictive to assume that $\Gamma_{t, x}$ is an $(M+1)$-cone, i.e. it has the form (2.4) with $N=M+1$. By the compactness of $S$ we can extract a finite subcovering $\left\{\Gamma^{i} ; 1 \leq i \leq \nu\right\}$, with $\Gamma_{i} \doteq \Gamma_{t_{i}, x_{i}}$. Define $\Delta^{i} \doteq \Gamma_{i} \backslash \bigcup_{j<i} \Gamma_{j}$ and set $\theta_{j}^{i}=\theta_{j}\left(t_{i}, x_{i}\right), y_{j}^{i}=y_{j}\left(t_{i}, x_{i}\right), \psi_{j}^{i}=\psi_{j}^{\left(t_{i}, x_{i}\right)}, \varphi_{j}^{i}=\varphi_{j}{ }^{\left(t_{i}, x_{i}\right)}$. Choose 
an integer $N$ such that

$$
N>\frac{8 M \nu^{2} T}{\varepsilon}
$$

and divide $[0, T]$ into $N$ equal subintervals $J_{1}, \ldots, J_{N}$, with

$$
J_{k}=\left[t_{k-1}, t_{k}\right), \quad t_{k}=\frac{k T}{N} .
$$

For each $i, k$ such that $\left(J_{k} \times \mathbb{R}^{n}\right) \cap \Delta^{i} \neq \emptyset$, we then split $J_{k}$ into $n+1$ subintervals $J_{k, 0}^{i}, \ldots, J_{k, n}^{i}$ with lengths proportional to $\theta_{0}^{i}, \ldots, \theta_{n}^{i}$, by setting

$$
J_{k, j}^{i}=\left[t_{k, j-1}, t_{k, j}\right), \quad t_{k, j}=\frac{T}{N}\left(k+\sum_{l=0}^{j} \theta_{l}^{i}\right), \quad t_{k,-1}=\frac{T k}{N} .
$$

For any point $(t, x) \in \overline{\Delta^{i}}$ we now set

$$
\left\{\begin{array}{l}
g^{i}(t, x) \doteq \psi_{j}^{i}(t, x) \\
\bar{g}^{i}(t, x)=y_{j}^{i}
\end{array} \quad \text { if } t \in \bigcup_{k=1}^{N} J_{k, j}^{i} .\right.
$$

The piecewise Lipschitz selection $g$ and a piecewise constant approximation $\bar{g}$ of $g$ can now be defined as

$$
g=\sum_{i=1}^{\nu} g^{i} \chi_{\Delta^{i}}, \quad \bar{g}=\sum_{i=1}^{\nu} \bar{g}^{i} \chi_{\Delta^{i}}
$$

By construction, recalling (3.7) and (3.8), the conditions (a), (b) in (i) clearly hold.

It remains to show that the estimates in (ii) hold as well. Let $\tau, \tau^{\prime} \in[0, T]$ and $u \in Y$ be such that $(t, u(t)) \in S$ for every $t \in\left[\tau, \tau^{\prime}\right]$, and define

$$
E^{i}=\left\{t \in I ;(t, u(t)) \in \Delta^{i}\right\}, \quad i=1, \ldots, \nu .
$$

From our previous definition $\Delta^{i} \doteq \Gamma_{i} \backslash \bigcup_{j<i} \Gamma_{j}$, where each $\Gamma_{j}$ is an $(M+1)$ cone, it follows that every $E^{i}$ is the union of at most $i$ disjoint intervals. We can thus write

$$
E^{i}=\left(\bigcup_{J_{k} \subset E^{i}} J_{k}\right) \cup \widehat{E}^{i}
$$

with $J_{k}$ given by $(3.11)$ and

$$
m\left(\widehat{E}^{i}\right) \leq \frac{2 i T}{N} \leq \frac{2 \nu T}{N} .
$$

Since

$$
\phi\left(t_{i}, x_{i}\right)=\sum_{j=0}^{n} \theta_{j}^{i} y_{j}^{i}
$$


the definition of $\bar{g}$ in (3.12), (3.13) implies

$$
\int_{J_{k}}\left[\phi\left(t_{i}, x_{i}\right)-\bar{g}(s, u(s))\right] d s=m\left(J_{k}\right)\left[\phi\left(t_{i}, x_{i}\right)-\sum_{j=0}^{n} \theta_{j}^{i} y_{j}^{i}\right]=0 .
$$

Therefore, from (3.9) and (3.6) it follows that

$$
\begin{aligned}
& \left|\int_{J_{k}}[\phi(s, u(s))-g(s, u(s))] d s\right| \\
& \leq\left|\int_{J_{k}}\left[\phi(s, u(s))-\phi\left(t_{i}, x_{i}\right)\right] d s\right| \\
& \quad+\left|\int_{J_{k}}\left[\phi\left(t_{i}, x_{i}\right)-\bar{g}(s, u(s))\right] d s\right|+\left|\int_{J_{k}}[\bar{g}(s, u(s))-g(s, u(s))] d s\right| \\
& \leq m\left(J_{k}\right)\left[\frac{\varepsilon}{4 T}+0+\frac{\varepsilon}{4 T}\right]=m\left(J_{k}\right) \frac{\varepsilon}{2 T} .
\end{aligned}
$$

The choice of $N$ in (3.10) and the bound (3.14) thus imply

$$
\begin{aligned}
\left|\int_{\tau}^{\tau^{\prime}}[\phi(s, u(s))-g(s, u(s))] d s\right| & \leq 2 M m\left(\bigcup_{i=1}^{\nu} \widehat{E}^{i}\right)+\left(\tau^{\prime}-\tau\right) \frac{\varepsilon}{2 T} \\
& \leq 2 M \nu \frac{2 \nu T}{N}+\frac{\varepsilon}{2} \leq \varepsilon,
\end{aligned}
$$

proving (3.4).

We next consider (3.5). For a fixed $i \in\{1, \ldots, \nu\}$, let $E^{i}$ be as before and define

$$
\xi_{-1}=0, \quad \xi_{j}=\sum_{l=0}^{j} \theta_{l}^{i}, \quad w^{i}(\xi)=\sum_{j=0}^{n} y_{j}^{i} \chi_{\left[\xi_{j-1}, \xi_{j}\right]} .
$$

Recalling (3.15), the definition of $h$ at (2.1) and Hölder's inequality together imply

$$
\begin{aligned}
h\left(\phi\left(t_{i}, x_{i}\right), F\left(t_{i}, x_{i}\right)\right) & \geq\left(\int_{0}^{1}\left|\phi\left(t_{i}, x_{i}\right)-w^{i}(\xi)\right|^{2} d \xi\right)^{1 / 2} \\
& \geq \int_{0}^{1}\left|\phi\left(t_{i}, x_{i}\right)-w^{i}(\xi)\right| d \xi \\
& =\sum_{j=0}^{n} \theta_{j}^{i}\left|\phi\left(t_{i}, x_{i}\right)-y_{j}^{i}\right| .
\end{aligned}
$$


Using this inequality we obtain

$$
\begin{aligned}
\int_{J_{k}}\left|\phi\left(t_{i}, x_{i}\right)-\bar{g}(s, u(s))\right| d s & =m\left(J_{k}\right) \sum_{j=0}^{n} \theta_{j}^{i}\left|\phi\left(t_{i}, x_{i}\right)-y_{j}^{i}\right| \\
& \leq m\left(J_{k}\right) \cdot h\left(\phi\left(t_{i}, x_{i}\right), F\left(t_{i}, x_{i}\right)\right) \leq \eta m\left(J_{k}\right),
\end{aligned}
$$

and therefore, by (3.9) and (3.6),

$$
\begin{aligned}
& \int_{J_{k}}|\phi(s, u(s))-g(s, u(s))| d s \\
& \leq \int_{J_{k}}\left|\phi(s, u(s))-\phi\left(t_{i}, x_{i}\right)\right| d s+\int_{J_{k}}|\bar{g}(s, u(s))-g(s, u(s))| d s \\
& \quad+\int_{J_{k}}\left|\phi\left(t_{i}, x_{i}\right)-\bar{g}(s, u(s))\right| \\
& \leq m\left(J_{k}\right)\left[\frac{\varepsilon}{4 T}+\frac{\varepsilon}{4 T}+\eta\right]=m\left(J_{k}\right)\left(\frac{\varepsilon}{2 T}+\eta\right) .
\end{aligned}
$$

Using again (3.14) and (3.10), we conclude that

$$
\begin{aligned}
\int_{\tau}^{\tau^{\prime}}|\phi(s, u(s))-g(s, u(s))| d s & \leq\left(\tau^{\prime}-\tau\right)\left(\frac{\varepsilon}{2 T}+\eta\right)+2 M \nu \frac{2 \nu T}{N} \\
& \leq \varepsilon+\left(\tau^{\prime}-\tau\right) \eta
\end{aligned}
$$

which finishes the proof of Lemma 3.

Using Lemma 3, given any continuous selection $\tilde{f}$ of $F$ on $\Omega^{\dagger}$, and any sequence $\left(\varepsilon_{k}\right)_{k \geq 1}$ of strictly positive numbers, we can generate a sequence $\left(f_{k}\right)_{k \geq 1}$ of selections from $F$ as follows.

To construct $f_{1}$, we apply the lemma with $S=\Omega^{\dagger}, \phi=f_{0}, \varepsilon=\varepsilon_{1}$. This yields a partition $\left\{A_{1}^{i} ; i=1, \ldots, \nu_{1}\right\}$ of $\Omega^{\dagger}$ and a piecewise Lipschitz selection $f_{1}$ of $F$ of the form

$$
f_{1}=\sum_{i=1}^{\nu_{1}} f_{1}^{i} \chi_{A_{1}^{i}}
$$

In general, at the beginning of the $k$ th step we are given a partition of $\Omega^{\dagger}$, say $\left\{A_{k}^{i} ; i=1, \ldots, \nu_{k}\right\}$, and a selection

$$
f_{k}=\sum_{i=1}^{\nu_{k}} f_{k}^{i} \chi_{A_{k}^{i}},
$$

where each $f_{k}^{i}$ is Lipschitz continuous and satisfies

$$
h\left(f_{k}(t, x), F(t, x)\right) \leq \varepsilon_{k} \quad \forall(t, x) \in \overline{A_{k}^{i}} .
$$


We then apply Lemma 3 separately to each $A_{k}^{i}$, choosing $S=\overline{A_{k}^{i}}, \varepsilon=\varepsilon_{k}$, $\phi=f_{k}^{i}$. This yields a partition $\left\{A_{k+1}^{i} ; i=1, \ldots, \nu_{k+1}\right\}$ of $\Omega^{\dagger}$ and functions of the form

$$
f_{k+1}=\sum_{i=1}^{\nu_{k+1}} f_{k+1}^{i} \chi_{A_{k+1}^{i}}, \quad \varphi_{k+1}^{i}(\cdot)=\left\langle a_{k+1}^{i}, \cdot\right\rangle+b_{k+1}^{i},
$$

where each $f_{k+1}^{i}: \overline{A_{k+1}^{i}} \rightarrow \mathbb{R}^{n}$ is a Lipschitz continuous selection from $F$, satisfying the following estimates:

$$
\begin{gathered}
\varphi_{k+1}^{i}(z)>h(z, F(t, x)) \quad \forall(t, x) \in A_{k+1}^{i}, \\
\varphi_{k+1}^{i}\left(f_{k+1}^{i}(t, x)\right) \leq \varepsilon_{k+1} \quad \forall(t, x) \in A_{k+1}^{i}, \\
\left|\int_{\tau}^{\tau^{\prime}}\left[f_{k+1}(s, u(s))-f_{k}(s, u(s))\right] d s\right| \leq \varepsilon_{k+1}, \\
\int_{\tau}^{\tau^{\prime}}\left|f_{k+1}(s, u(s))-f_{k}(s, u(s))\right| d s \leq \varepsilon_{k+1}+\varepsilon_{k}\left(\tau^{\prime}-\tau\right),
\end{gathered}
$$

for every $u \in Y$ and every $\tau, \tau^{\prime}$, as long as the values $(s, u(s))$ remain inside a single set $A_{k}^{i}$, for $s \in\left[\tau, \tau^{\prime}\right)$.

Observe that, according to Lemma 3 , each $A_{k}^{i}$ is closed-open in the finer topology generated by all $(M+1)$-cones. Therefore, each $f_{k}$ is $\Gamma^{M+1}$ continuous. By Theorem 2 in [2], the substitution operator $\mathcal{S}^{f_{k}}: u(\cdot) \mapsto$ $f_{k}(\cdot, u(\cdot))$ is continuous from the set $Y$ defined in $(2.5)$ into $\mathcal{L}^{1}\left([0, T] ; \mathbb{R}^{n}\right)$. The Picard map $\mathcal{P}^{f_{k}}$ is thus continuous as well.

Furthermore, there exists an integer $N_{k}$ with the following property. Given any $u \in Y$, there exists a finite partition of $[0, T]$ with nodes $0=\tau_{0}<$ $\tau_{1}<\ldots<\tau_{n(u)}=T$, with $n(u) \leq N_{k}$, such that, as $t$ ranges in any $\left[\tau_{l-1}, \tau_{l}\right)$, the point $(t, u(t))$ remains inside one single set $A_{k}^{i}$. Otherwise stated, the number of times the curve $t \mapsto(t, u(t))$ crosses a boundary between two distinct sets $A_{k}^{i}, A_{k}^{j}$ is smaller than $N_{k}$, for every $u \in Y$. The construction of the $A_{k}^{i}$ in terms of $(M+1)$-cones implies that all these crossings are transversal. Since the restriction of $f_{k}$ to each $A_{k}^{i}$ is Lipschitz continuous, it is clear that every Cauchy problem

$$
\dot{x}(t)=f_{k}(t, x(t)), \quad x\left(t_{0}\right)=x_{0},
$$

has a unique solution, depending continuously on the initial data $\left(t_{0}, x_{0}\right) \in$ $[0, T] \times D$.

From (3.18), (3.19) and the property of $N_{k}$ it follows that

$$
\begin{aligned}
& \left|\int_{0}^{t}\left[f_{k+1}(s, u(s))-f_{k}(s, u(s))\right] d s\right| \\
& \quad \leq \sum_{l=1}^{L}\left|\int_{\tau_{l-1}}^{\tau_{l}}\left[f_{k+1}(s, u(s))-f_{k}(s, u(s))\right] d s\right| \leq N_{k} \varepsilon_{k+1}
\end{aligned}
$$


where $0=\tau_{0}<\tau_{1}<\ldots<\tau_{L}=t$ are the times at which the map $s \mapsto$ $(s, u(s))$ crosses a boundary between two distinct sets $A_{k}^{i}, A_{k}^{j}$. Since (3.20) holds for every $t \in[0, T]$, we conclude that

$$
\left\|\mathcal{P}^{f_{k+1}}-\mathcal{P}^{f_{k}}\right\| \leq N_{k} \varepsilon_{k+1} .
$$

Similarly, for every $u \in Y$ one has

$$
\begin{aligned}
& \left\|f_{k+1}(\cdot, u(\cdot))-f_{k}(\cdot, u(\cdot))\right\|_{\mathcal{L}^{1}\left([0, T] ; \mathbb{R}^{n}\right)} \\
& \quad \leq \sum_{l=1}^{n(u)} \int_{\tau_{l-1}}^{\tau_{l}}\left|f_{k+1}(s, u(s))-f_{k}(s, u(s))\right| d s \\
& \quad \leq \sum_{l=1}^{n(u)}\left[\varepsilon_{k+1}+\varepsilon_{k}\left(\tau_{l}-\tau_{l-1}\right)\right] \leq N_{k} \varepsilon_{k+1}+\varepsilon_{k} T .
\end{aligned}
$$

Now consider the functions $\varphi_{k}: \mathbb{R}^{n} \times \Omega^{\dagger} \rightarrow \mathbb{R}$ with

$$
\varphi_{k}(y, t, x) \doteq\left\langle a_{k}^{i}, y\right\rangle+b_{k}^{i} \quad \text { if }(t, x) \in A_{k}^{i} .
$$

From (3.16), (3.17) it follows that

$$
\begin{aligned}
\varphi_{k}(y, t, x) \geq h(y, F(t, x)) & \forall(t, x) \in \Omega^{\dagger}, y \in F(t, x), \\
\varphi_{k}\left(f_{k}(t, x), t, x\right) \leq \varepsilon_{k} & \forall(t, x) \in \Omega^{\dagger} .
\end{aligned}
$$

For every $u \in Y,(3.18)$ and the linearity of $\varphi_{k}$ in $y$ imply

$$
\begin{aligned}
& \left|\int_{0}^{T}\left[\varphi_{k}\left(f_{k+1}(s, u(s)), s, u(s)\right)-\varphi_{k}\left(f_{k}(s, u(s)), s, u(s)\right)\right] d s\right| \\
& \quad \leq \sum_{l=1}^{n(u)} \max \left\{\left|a_{k}^{1}\right|, \ldots,\left|a_{k}^{\nu_{k}}\right|\right\}\left|\int_{\tau_{l-1}}^{\tau_{l}}\left[f_{k+1}(s, u(s))-f_{k}(s, u(s))\right] d s\right| \\
& \quad \leq N_{k} \max \left\{\left|a_{k}^{1}\right|, \ldots,\left|a_{k}^{\nu_{k}}\right|\right\} \varepsilon_{k+1} .
\end{aligned}
$$

Moreover, for every $l \geq k$, from (3.19) it follows that

$$
\begin{aligned}
& \int_{0}^{T} \mid \varphi_{k}\left(f_{l+1}(s, u(s)), s, u(s)\right)-\varphi_{k}\left(f_{l}(s, u(s)), s, u(s)\right) \mid d s \\
& \leq \max \left\{\left|a_{k}^{1}\right|, \ldots,\left|a_{k}^{\nu_{k}}\right|\right\} \int_{0}^{T}\left|f_{l+1}(s, u(s))-f_{l}(s, u(s))\right| d s \\
& \leq \max \left\{\left|a_{k}^{1}\right|, \ldots,\left|a_{k}^{\nu_{k}}\right|\right\} \cdot\left(N_{l} \varepsilon_{l+1}+\varepsilon_{l} T\right) .
\end{aligned}
$$

Observe that all of the above estimates hold regardless of the choice of the $\varepsilon_{k}$. We now introduce an inductive procedure for choosing the constants $\varepsilon_{k}$, 
which will yield the convergence of the sequence $f_{k}$ to a function $f$ with the desired properties.

Given $f_{0}$ and $\varepsilon_{0}$, by Lemma 2 there exists $\delta_{0}>0$ such that, if $g: \Omega^{\dagger} \rightarrow$ $\bar{B}(0, M)$ and $\left\|\mathcal{P}^{g}-\mathcal{P}^{f_{0}}\right\| \leq \delta_{0}$, then, for each $\left(t_{0}, x_{0}\right) \in[0, T] \times D$, every solution of (2.7) remains $\varepsilon_{0}$-close to the unique solution of (1.3). We then choose $\varepsilon_{1}=\delta_{0} / 2$.

By induction on $k$, assume that the functions $f_{1}, \ldots, f_{k}$ have been constructed, together with the linear functions $\varphi_{l}^{i}(\cdot)=\left\langle a_{l}^{i}, \cdot\right\rangle+b_{l}^{i}$ and the integers $N_{l}, l=1, \ldots, k$. Let the values $\delta_{0}, \delta_{1}, \ldots, \delta_{k}>0$ be inductively chosen, satisfying

$$
\delta_{l} \leq \delta_{l-1} / 2, \quad l=1, \ldots, k,
$$

and such that $\left\|\mathcal{P}^{g}-\mathcal{P}^{f_{l}}\right\| \leq \delta_{l}$ implies that for every $\left(t_{0}, x_{0}\right) \in[0, T] \times D$ the solution set of (2.7) has diameter $\leq 2^{-l}$, for $l=1, \ldots, k$. This is possible again because of Lemma 2 . For $k \geq 1$ we then choose

$$
\varepsilon_{k+1} \doteq \min \left\{\frac{\delta_{k}}{2 N_{k}}, \frac{2^{-k}}{N_{k}}, \frac{2^{-k}}{N_{k} \max \left\{\left|a_{l}^{i}\right| ; 1 \leq l \leq k, 1 \leq i \leq \nu_{l}\right\}}\right\} .
$$

Using (3.28), (3.29) in (3.21), with $N_{0} \doteq 1$, we now obtain

$$
\sum_{k=p}^{\infty}\left\|\mathcal{P}^{f_{k+1}}-\mathcal{P}^{f_{k}}\right\| \leq \sum_{k=p}^{\infty} N_{k} \frac{\delta_{k}}{2 N_{k}} \leq \sum_{k=p}^{\infty} \frac{2^{p-k} \delta_{p}}{2} \leq \delta_{p}
$$

for every $p \geq 0$. From (3.22) and (3.29) we further obtain

$$
\begin{aligned}
\sum_{k=1}^{\infty}\left\|f_{k+1}(\cdot, u(\cdot))-f_{k}(\cdot, u(\cdot))\right\|_{\mathcal{L}^{1}} & \leq \sum_{k=1}^{\infty}\left(N_{k} \frac{2^{-k}}{N_{k}}+\frac{2^{1-k} T}{N_{k}}\right) \\
& \leq \sum_{k=1}^{\infty}\left(2^{-k}+2^{1-k} T\right) \leq 1+2 T
\end{aligned}
$$

Define

$$
f(t, x) \doteq \lim _{k \rightarrow \infty} f_{k}(t, x)
$$

for all $(t, x) \in \Omega^{\dagger}$ at which the sequence $f_{k}$ converges. By (3.31), for every $u \in Y$ the sequence $f_{k}(\cdot, u(\cdot))$ converges in $\mathcal{L}^{1}\left([0, T] ; \mathbb{R}^{n}\right)$ and a.e. on $[0, T]$. In particular, considering the constant functions $u \equiv x \in \bar{B}(D, M T)$, by Fubini's theorem we conclude that $f$ is defined a.e. on $\Omega^{\dagger}$. Moreover, the substitution operators $\mathcal{S}^{f_{k}}: u(\cdot) \mapsto f_{k}(\cdot, u(\cdot))$ converge to the operator $\mathcal{S}^{f}: u(\cdot) \mapsto f(\cdot, u(\cdot))$ uniformly on $Y$. Since each $\mathcal{S}^{f_{k}}$ is continuous, $\mathcal{S}^{f}$ is also continuous. Clearly, the Picard map $\mathcal{P}^{f}$ is continuous as well. By (3.30) 
we have

$$
\left\|\mathcal{P}^{f}-\mathcal{P}^{f_{k}}\right\| \leq \sum_{k=p}^{\infty}\left\|\mathcal{P}^{f_{k+1}}-\mathcal{P}^{f_{k}}\right\| \leq \delta_{p} \quad \forall p \geq 1 .
$$

Recalling the property of $\delta_{p}$, this implies that, for every $p$, the solution set of (2.7) has diameter $\leq 2^{-p}$. Since $p$ is arbitrary, for every $\left(t_{0}, x_{0}\right) \in$ $[0, T] \times D$ the Cauchy problem can have at most one solution. On the other hand, the existence of such a solution is guaranteed by Schauder's theorem. The continuous dependence of this solution on the initial data $t_{0}, x_{0}$, in the norm of $\mathcal{A C}$, is now an immediate consequence of uniqueness and of the continuity of the operators $\mathcal{S}^{f}, \mathcal{P}^{f}$. Furthermore, for $p=0$, (3.30) yields $\left\|\mathcal{P}^{f}-\mathcal{P}^{f_{0}}\right\| \leq \delta_{0}$. The choice of $\delta_{0}$ thus implies (1.4).

It now remains to prove (1.1). Since every set $F(t, x)$ is closed, it is clear that $f(t, x) \in F(t, x)$. For every $u \in Y$ and $k \geq 1$, by (3.24)-(3.27) the choices of $\varepsilon_{k}$ at (3.29) yield

$$
\begin{aligned}
& \int_{0}^{T} h(f(s, u(s)), F(s, u(s))) d s \\
& \leq \int_{0}^{T} \varphi_{k}(f(s, u(s)), s, u(s)) d s \\
& \leq \int_{0}^{T} \varphi_{k}\left(f_{k}(s, u(s)), s, u(s)\right) d s \\
& \quad+\left|\int_{0}^{T}\left[\varphi_{k}\left(f_{k+1}(s, u(s)), s, u(s)\right)-\varphi_{k}\left(f_{k}(s, u(s)), s, u(s)\right)\right] d s\right| \\
& \quad+\sum_{l=k+1}^{\infty} \int_{0}^{T} \mid \varphi_{k}\left(f_{l+1}((s, u(s)), s, u(s))-\varphi_{k}\left(f_{l}(s, u(s)), s, u(s)\right) \mid d s\right. \\
& \leq 2^{1-k} T+2^{-k}+\sum_{l=k+1}^{\infty}\left(2^{-l}+2^{1-l} T\right) .
\end{aligned}
$$

Observing that the right hand side of (3.33) approaches zero as $k \rightarrow \infty$, we conclude that

$$
\int_{0}^{T} h(f(t, u(t)), F(t, u(t))) d t=0 .
$$

By (2.2), given any $u \in Y$, this implies $f(t, u(t)) \in \operatorname{ext} F(t, u(t))$ for almost every $t \in[0, T]$. By possibly redefining $f$ on a set of measure zero, this yields (1.1). 
4. Applications. Throughout this section we make the following assumptions:

(H) $\quad F:[0, T] \times \Omega \rightarrow \bar{B}(0, M)$ is a bounded continuous multifunction with compact values satisfying (LSP), while $D$ is a compact set such that $\bar{B}(D, M T) \subset \Omega$.

An immediate consequence of Theorem 1 is

COROllary 1. Let the hypotheses $(\mathrm{H})$ hold. Then there exists a continuous map $\left(t_{0}, x_{0}\right) \mapsto x\left(\cdot, t_{0}, x_{0}\right)$ from $[0, T] \times D$ into $\mathcal{A C}$ such that

$$
\begin{cases}\dot{x}\left(t, t_{0}, x_{0}\right) \in \operatorname{ext} F\left(t, x\left(t, t_{0}, x_{0}\right)\right) & \forall t \in[0, T], \\ x\left(t_{0}, t_{0}, x_{0}\right)=x_{0} & \forall t_{0}, x_{0} .\end{cases}
$$

Another consequence of Theorem 1 is the contractibility of the sets of solutions of certain differential inclusions. We recall here that a metric space $X$ is contractible if there exist a point $\widetilde{u} \in X$ and a continuous mapping $\Phi: X \times[0,1] \rightarrow X$ such that

$$
\Phi(v, 0)=\widetilde{u}, \quad \Phi(v, 1)=v, \quad \forall v \in X .
$$

The map $\Phi$ is then called a null homotopy of $X$.

COROllary 2. Let the assumptions $(\mathrm{H})$ hold. Then, for any $\bar{x} \in D$, the sets $\mathcal{M}, \mathcal{M}^{\text {ext }}$ of solutions of

$$
\begin{aligned}
& x(0)=\bar{x}, \quad \dot{x}(t) \in F(t, x(t)), \quad t \in[0, T], \\
& x(0)=\bar{x}, \quad \dot{x} \in \operatorname{ext} F(t, x(t)), \quad t \in[0, T],
\end{aligned}
$$

are both contractible in $\mathcal{A C}$.

Proof. Let $f$ be a selection from ext $F$ with the properties stated in Theorem 1. As usual, we denote by $x\left(\cdot, t_{0}, x_{0}\right)$ the unique solution of the Cauchy problem (1.2). Define the null homotopy $\Phi: \mathcal{M} \times[0,1] \rightarrow \mathcal{M}$ by

$$
\Phi(v, \lambda)(t) \doteq \begin{cases}v(t) & \text { if } t \in[0, \lambda T] \\ x(t, \lambda T, v(\lambda T)) & \text { if } t \in[\lambda T, T] .\end{cases}
$$

By Theorem $1, \Phi$ is continuous. Moreover, setting $\widetilde{u}(\cdot) \doteq u(\cdot, 0, \bar{x})$, we obtain

$$
\Phi(v, 0)=\widetilde{u}, \quad \Phi(v, 1)=v, \quad \Phi(v, \lambda) \in \mathcal{M} \quad \forall v \in \mathcal{M},
$$

proving that $\mathcal{M}$ is contractible. We now observe that, if $v \in \mathcal{M}^{\text {ext }}$, then $\Phi(v, \lambda) \in \mathcal{M}^{\text {ext }}$ for every $\lambda$. Therefore, $\mathcal{M}^{\text {ext }}$ is contractible as well.

Our last application is concerned with feedback controls. Let $\Omega \subseteq \mathbb{R}^{n}$ be open, $U \subset \mathbb{R}^{m}$ compact, and let $g:[0, T] \times \Omega \times U \rightarrow \mathbb{R}^{n}$ be a continuous function. By a well-known theorem of Filippov [11], the solutions of the control system

$$
\dot{x}=g(t, x, u), \quad u \in U,
$$


correspond to the trajectories of the differential inclusion

$$
\dot{x} \in F(t, x) \doteq\{g(t, x, \omega) ; \omega \in U\} .
$$

In connection with (4.1), one can consider the "relaxed" system

$$
\dot{x}=g^{\#}\left(t, x, u^{\#}\right), \quad u^{\#} \in U^{\#},
$$

whose trajectories are precisely those of the differential inclusion

$$
\dot{x} \in F^{\#}(t, x) \doteq \overline{\mathrm{co}} F(t, x) .
$$

The control system (4.3) is obtained by defining the compact set

$$
U^{\#} \doteq U \times \ldots \times U \times \Delta_{n}=U^{n+1} \times \Delta_{n},
$$

where

$$
\Delta_{n} \doteq\left\{\theta=\left(\theta_{0}, \ldots, \theta_{n}\right) ; \sum_{i=0}^{n} \theta_{i}=1, \theta_{i} \geq 0 \forall i\right\}
$$

is the standard simplex in $\mathbb{R}^{n+1}$, and by setting

$$
g^{\#}\left(t, x, u^{\#}\right)=g^{\#}\left(t, x,\left(u_{0}, \ldots, u_{n},\left(\theta_{0}, \ldots, \theta_{n}\right)\right)\right) \doteq \sum_{i=0}^{n} \theta_{i} f\left(t, x, u_{i}\right) .
$$

Generalized controls of the form $u^{\#}=\left(u_{0}, \ldots, u_{n}, \theta\right)$ taking values in the set $U^{n+1} \times \Delta_{n}$ are called chattering controls.

Corollary 3. Consider the control system (4.1), with $g:[0, T] \times \Omega \times$ $U \rightarrow \bar{B}(0, M)$ Lipschitz continuous. Let $D$ be a compact set with $\bar{B}(D ; M T)$ $\subset \Omega$. Let $u^{\#}(t, x) \in U^{\#}$ be a chattering feedback control such that the mapping

$$
(t, x) \mapsto g^{\#}\left(t, x, u^{\#}(t, x)\right) \doteq f_{0}(t, x)
$$

is Lipschitz continuous.

Then for every $\varepsilon_{0}>0$ there exists a measurable feedback control $\bar{u}=$ $\bar{u}(t, x)$ with the following properties:

(a) for every $(t, x)$, one has $g(t, x, \bar{u}(t, x)) \in \operatorname{ext} F(t, x)$, with $F$ as in (4.2),

(b) for every $\left(t_{0}, x_{0}\right) \in[0, T] \times D$, the Cauchy problem

$$
\dot{x}(t)=g(t, x(t), \bar{u}(t, x(t))), \quad x\left(t_{0}\right)=x_{0},
$$

has a unique solution $x\left(\cdot, t_{0}, x_{0}\right)$,

(c) if $y\left(\cdot, t_{0}, x_{0}\right)$ denotes the (unique) solution of the Cauchy problem

$$
\dot{y}=f_{0}(t, y(t)), \quad y\left(t_{0}\right)=x_{0},
$$

then for every $\left(t_{0}, x_{0}\right)$ one has

$$
\left|x\left(t, t_{0}, x_{0}\right)-y\left(t, t_{0}, x_{0}\right)\right|<\varepsilon_{0} \quad \forall t \in[0, T] .
$$

Proof. The Lipschitz continuity of $g$ implies that the multifunction $F$ in (4.2) is Lipschitz continuous in the Hausdorff metric, hence it satisfies 
(LSP). We can thus apply Theorem 1 , and obtain a suitable selection $f$ of ext $F$, in connection with $f_{0}, \varepsilon_{0}$. For every $(t, x)$, the set

$$
W(t, x) \doteq\{\omega \in U ; g(t, x, \omega)=f(t, x)\} \subset \mathbb{R}^{m}
$$

is a compact nonempty subset of $U$. Let $\bar{u}(t, x) \in W(t, x)$ be the lexicographic selection. Then the feedback control $\bar{u}$ is measurable, and it is trivial to check that $\bar{u}$ has all the required properties.

\section{References}

[1] J. P. A ubin and A. Cellina, Differential Inclusions, Springer, Berlin, 1984.

[2] A. Bressan, Directionally continuous selections and differential inclusions, Funkcial. Ekvac. 31 (1988), 459-470.

[3] - On the qualitative theory of lower semicontinuous differential inclusions, J. Differential Equations 77 (1989), 379-391.

[4] —, The most likely path of a differential inclusion, ibid. 88 (1990), 155-174.

[5] - Selections of Lipschitz multifunctions generating a continuous flow, Differential Integral Equations 4 (1991), 483-490.

[6] A. Bressan and G. Colombo, Boundary value problems for lower semicontinuous differential inclusions, Funkcial. Ekvac. 36 (1993), 359-373.

[7] A. Cellina, On the set of solutions to Lipschitzean differential inclusions, Differential Integral Equations 1 (1988), 495-500.

[8] F. S. De Blasi and G. Pianigiani, On the solution set of nonconvex differential inclusions, J. Differential Equations, to appear.

[9] - - - Topological properties of nonconvex differential inclusions, Nonlinear Anal. 20 (1993), 871-894.

[10] A. LeDonne and M. V. Marchi, Representation of Lipschitz compact convex valued mappings, Atti Accad. Naz. Lincei Rend. 68 (1980), 278-280.

[11] A. F. Filippov, On certain questions in the theory of optimal control, SIAM J. Control Optim. 1 (1962), 76-84.

[12] A. Ornelas, Parametrization of Carathéodory multifunctions, Rend. Sem. Mat. Univ. Padova 83 (1990), 33-44.

[13] A. A. Tolstonogov, Extreme continuous selectors of multivalued maps and their applications, preprint, 1992.

S.I.S.S.A.

VIA BEIRUT 4

TRIESTE 34014

ITALY 